\title{
Adaptive Neuron-Like Control of Time-Delay Systems Enhanced with Feedforward and Supervisory Strategies
}

\author{
Yau-Zen Chang, ${ }^{1}$ Jung-Fu Hou, ${ }^{1}$ and Zhi-Ren Tsai ${ }^{2,3}$ \\ ${ }^{1}$ Department of Mechanical Engineering, Chang Gung University, Tao-Yuan 33302, Taiwan \\ ${ }^{2}$ Department of Computer Science \& Information Engineering, Asia University, Wufeng, Taichung 41354, Taiwan \\ ${ }^{3}$ Graduate Institute of Biostatistics, China Medical University, 91 Hsueh-Shih Road, Taichung 404, Taiwan
}

Correspondence should be addressed to Yau-Zen Chang; zen@mail.cgu.edu.tw

Received 15 April 2013; Accepted 18 June 2013

Academic Editor: Qingsong Xu

Copyright (C) 2013 Yau-Zen Chang et al. This is an open access article distributed under the Creative Commons Attribution License, which permits unrestricted use, distribution, and reproduction in any medium, provided the original work is properly cited.

\begin{abstract}
Tracking control of nonlinear systems with significant delay effects has been the focus of intensive research. In this paper, we propose an effective supervised adaptive control scheme to tackle the problem. The scheme is composed of an adaptive control part of two neuron-like models with delay effects and a supervisory control part to enhance robustness against disturbance and model uncertainties. A design methodology based on the Lyapunov analysis is presented. Experimental results obtained from a practical temperature control system show that not only is the design procedure conceptually simple but also the control performance is also excellent when compared with the traditional PD controller. Also, the feedforward term is able to provide extra improvement in the regulation performance.
\end{abstract}

\section{Introduction}

The study of stability and stabilization for time-delay systems has received considerable attention in recent years [1-4] since delay is a major source of instability in many important engineering systems $[5,6]$. For instance, Hopf bifurcation caused by time delay is extensively investigated in [7-10].

The applicability of neural-network-based techniques in nonlinear control systems has been successively demonstrated in [11-13] because of their unique modeling capability and adaptability $[14,15]$. However, delay effects are not effectively considered in most of the proposed schemes and modeling error is ignored, which may be a potential source of instability [16]. As neural networks have superior capability in the construction of models of complex nonlinear systems, [17-20] use a feed-forward neural network for model-based predictive control. However, only simulation results are demonstrated in most of these researches. Reference [21] also applied an indirectly derived feedforward term in a simulation study, but the approach is based on predictability of disturbances.

In this paper, a particular class of adaptive neural controller is proposed based on a time-delay neural model.
Inspired by [22], the model-based adaptation law has two auto-tuning neurons in which both delay effects and feedforward terms are explicitly included, which are not considered in the original contribution. Robustness and stability conditions are derived in the sense of Lyapunov for the design of the proposed adaptation scheme, and performance of the proposed scheme is demonstrated by experimental results of a temperature control system.

\section{Problem Formulation}

The plant under control is assumed to be nonlinear with system dynamics being represented as

$$
x^{(n)}(t)=f\left(X, X_{d}, w_{m}(t, \tau)\right)+g(X) u(t)+d_{0}(t),
$$

where $u(t)$ is the control input, $d_{0}$ is unknown but bounded disturbance with $\left|d_{0}(t)\right| \leq d_{U}$, and $w_{m}(t, \tau)$ is measurable or predictable disturbance with delay $\tau$. Furthermore, $X=$ $\left[\xi(t), \dot{\xi}(t), \ldots, \xi^{(n-1)}(t)\right]^{T}=\left[x_{1}(t), x_{2}(t), \ldots, x_{n}(t)\right]^{T} \in \Re^{n \times 1}$ is the state vector of the nonlinear system with $x(t)$ being the state of the system and $x_{i}(t), i=1 \sim n$ being its derivatives, and $X_{d}=\left[\xi\left(t-\tau_{d}\right), \dot{\xi}\left(t-\tau_{d}\right), \ldots, \xi^{(n-1)}\left(t-\tau_{d}\right)\right]^{T}=\left[x_{1}\left(t-\tau_{d}\right)\right.$, 
$\left.x_{2}\left(t-\tau_{d}\right), \ldots, x_{n}\left(t-\tau_{d}\right)\right]^{T}$ is the delayed state vector with delay $\tau_{d} ; f(\cdot)$ is the nonlinear system function in $X, X_{d}$, and $w_{m}$. Note that the nonlinear input gain $g(\cdot)$ is assumed to be a function in $X$ only.

\section{Controller Design}

Firstly, we define the desired output as $y_{r}$ and tracking error ase $=y_{r}-\xi$. Then we may define $Y_{r}=\left[y_{r}, \dot{y}_{r}, \ldots, y_{r}^{(n-1)}\right]^{T}$ and $E=\left[e, \dot{e}, \ldots, e^{(n-1)}\right]^{T}$. Suppose that we choose a gain vector $K=\left[k_{0}, k_{1}, \ldots, k_{n-1}\right]^{T}$ such that all roots of $s^{n}+k_{n-1} s^{n-1}+$ $\cdots+k_{1} s+k_{0}=0$ are in the open left-half complex plane. The proposed control law $u(t)$ is given by

$$
u(t)=u_{C}\left(\theta_{f}, \theta_{g}\right)+u_{S},
$$

where $u_{C}\left(\theta_{f}, \theta_{g}\right)$ is an adaptive control law and $u_{S}$ is a supervisory control law which enhances the robustness of the closed-loop system and improves transient performance by keeping system states stay in some prespecified region. The adaptive control law is defined as

$$
u_{C}\left(\theta_{f}, \theta_{g}\right)=\widehat{g}^{-1}\left(\theta_{g}\right)\left[-\widehat{f}\left(\theta_{f}\right)+y_{r}^{(n)}+K^{T} E\right],
$$

where $\widehat{f}\left(\theta_{f}\right)$ and $\widehat{g}\left(\theta_{g}\right)$ are two neuron-like models:

$$
\begin{gathered}
\hat{g}\left(\theta_{g}\right)=\frac{2 a_{g}}{1+\exp \left(-b_{g} \cdot\left[-h_{g}+\sum_{i=1}^{n} \mu_{i} x_{i}(t)\right]\right)}, \quad(4) \\
\widehat{f}\left(\theta_{f}\right)=\left(a_{f} \cdot\{1-\exp \right. \\
\times\left(-b_{f} \cdot\left\{h_{f}+\sum_{j=0 i=1}^{J} \sum_{i j}^{n}\left[\mu_{i j} x_{i}(t-j \cdot \tau)\right]\right.\right. \\
\left.\left.\left.\times\left(1+\exp \quad+\sum_{l=0}^{L} K_{F l} w_{m}(t-l \cdot \tau)\right\}\right)\right\}\right) \\
\times\left(-b_{f} \cdot\left\{h_{f}+\sum_{j=0}^{J} \sum_{i=1}^{n}\left[\mu_{i j} x_{i}(t-j \cdot \tau)\right]\right.\right. \\
\left.\left.\left.+\sum_{l=0}^{L} K_{F l} w_{m}(t-l \cdot \tau)\right\}\right)\right)^{-1},
\end{gathered}
$$

where $\theta_{f}=\left[a_{f}, b_{f}, h_{f}, K_{F 0}, K_{F 1}, \ldots, K_{F L}, \mu_{10}, \mu_{20}, \ldots\right]^{T}$ and $\theta_{g}=\left[a_{g}, b_{g}, h_{g}, \mu_{1}, \mu_{2}, \ldots\right]^{T}$, with $a_{g}, b_{g}$, and $h_{g}$ being positive, are adjustable parameters, $w_{m}(t)$ is a feed-forward term, and $K_{F 0}, K_{F 1}, \ldots, K_{F L}$ are the corresponding feed-forward gains. In this study, two adaptation laws with projection mapping are defined as follows:

$$
\begin{gathered}
\dot{\theta}_{f}= \begin{cases}\varphi_{f}, & \text { if }\left(\left\|\theta_{f}\right\|<M_{f}\right) \\
\varphi_{f}-\frac{\theta_{f}}{\left\|\theta_{f}\right\|^{2}} \theta_{f}^{T} \varphi_{f}, & \text { or }\left(\left\|\theta_{f}\right\|=M_{f},-\theta_{f}^{T} \varphi_{f} \geq 0\right),\end{cases} \\
\dot{\theta}_{g}= \begin{cases}\varphi_{g}, & \text { if }\left(\left\|\theta_{g}\right\|<M_{g}\right) \\
\varphi_{g}-\frac{\theta_{g}}{\left\|\theta_{g}\right\|^{2}} \theta_{g}^{T} \varphi_{g}, & \text { otherwise, }\left(\left\|\theta_{g}\right\|=M_{g},-\theta_{g}^{T} \varphi_{g} \geq 0\right),\end{cases}
\end{gathered}
$$

where

$$
\begin{aligned}
& \varphi_{f}=-\gamma_{f} E^{T} P \cdot B_{c} \frac{\partial \widehat{f}}{\partial \theta_{f}}, \quad \gamma_{f}>0, \\
& \varphi_{g}=-\gamma_{g} E^{T} P \cdot B_{c} \frac{\partial \widehat{g}}{\partial \theta_{g}} u_{C}, \quad \gamma_{g}>0 ; \\
& \frac{\partial \widehat{f}}{\partial \theta_{f}}=\left[\frac{\partial \hat{f}}{\partial a_{f}}, \frac{\partial \hat{f}}{\partial b_{f}}, \frac{\partial \hat{f}}{\partial h_{f}}, \frac{\partial \hat{f}}{\partial K_{F 0}},\right. \\
& \left.\frac{\partial \widehat{f}}{\partial K_{F 1}}, \ldots, \frac{\partial \widehat{f}}{\partial K_{F L}}, \frac{\partial \widehat{f}}{\partial \mu_{10}}, \frac{\partial \widehat{f}}{\partial \mu_{20}}, \ldots\right]^{T}, \\
& \frac{\partial \hat{f}}{\partial a_{f}}=\frac{\widehat{f}}{a_{f}}, \quad \frac{\partial \widehat{g}}{\partial h_{g}}=-b_{g} \widehat{g} \cdot\left\{1-\frac{\widehat{g}}{2 a_{g}}\right\}, \\
& \frac{\partial \widehat{f}}{\partial b_{f}}=\frac{a_{f}\left\{h_{f}+\sum_{j=0}^{J} \sum_{i=1}^{n}\left[\mu_{i j} x_{i}(t-j \cdot \tau)\right]\right\}}{2}\left\{1+\frac{\hat{f}}{a_{f}}\right\} \\
& \times\left\{1-\frac{\widehat{f}}{a_{f}}\right\}, \\
& \frac{\partial \hat{f}}{\partial h_{f}}=\frac{a_{f} b_{f}}{2}\left\{1+\frac{\hat{f}}{a_{f}}\right\}\left\{1-\frac{\hat{f}}{a_{f}}\right\}, \\
& \frac{\partial \widehat{f}}{\partial \mu_{i j}}=\frac{a_{f} b_{f} x_{i}(t-j \cdot \tau)}{2}\left\{1+\frac{\widehat{f}}{a_{f}}\right\}\left\{1-\frac{\widehat{f}}{a_{f}}\right\}, \\
& \frac{\partial \hat{f}}{\partial K_{F l}}=\frac{a_{f} b_{f} d_{f f}(t-l \cdot \tau)}{2}\left\{1+\frac{\hat{f}}{a_{f}}\right\}\left\{1-\frac{\hat{f}}{a_{f}}\right\}, \\
& \frac{\partial \widehat{g}}{\partial a_{g}}=\frac{\widehat{g}}{a_{g}},
\end{aligned}
$$




$$
\begin{gathered}
\frac{\partial \widehat{g}}{\partial \theta_{g}}=\left[\frac{\partial \widehat{g}}{\partial a_{g}}, \frac{\partial \widehat{g}}{\partial b_{g}}, \frac{\partial \widehat{g}}{\partial h_{g}}, \frac{\partial \widehat{g}}{\partial \mu_{1}}, \frac{\partial \widehat{g}}{\partial \mu_{2}}, \ldots\right]^{T}, \\
\frac{\partial \widehat{g}}{\partial b_{g}}=\left[-h_{g}+\sum_{i=1}^{n} \mu_{i} x_{i}(t)\right] \hat{g} \cdot\left\{1-\frac{\widehat{g}}{2 a_{g}}\right\}, \\
\frac{\partial \widehat{g}}{\partial \mu_{i}}=b_{g} x_{i}(t) \hat{g} \cdot\left\{1-\frac{\widehat{g}}{2 a_{g}}\right\} .
\end{gathered}
$$

The adaptation law, (4) and (5), is designed to ensure the boundedness of $\theta_{f}$ and $\theta_{g}$. Substituting (2) into (1), we have

$$
\begin{aligned}
x^{(n)}= & f+g \cdot\left(u_{C}+u_{S}\right)+d_{0}=y_{r}^{(n)}+K^{T} E \\
& +[f-\hat{f}]+[g-\hat{g}] u_{C}+g \cdot u_{S}+d_{0} .
\end{aligned}
$$

This implies that

$$
e^{(n)}=-K^{T} E+\widehat{f}-f+[\widehat{g}-g] u_{C}-g \cdot u_{S}-d_{0} .
$$

Let $A_{c}=\left[\begin{array}{c}0_{(n-1) \times 1}, I_{(n-1) \times(n-1)} \\ -K^{T}\end{array}\right.$ and $B_{c}=\left[0_{1 \times(n-1)}, 1\right]^{T}$ be a companion form pair; we may rewrite (10) as

$$
\dot{E}=A_{c} E+B_{c} \cdot\left\{\widehat{f}-f+[\widehat{g}-g] u_{C}-g \cdot u_{S}-d_{0}\right\} .
$$

Now consider a Lyapunov function candidate

$$
V_{E}=2^{-1} E^{T} P \cdot E,
$$

where $P>0$ which satisfies the Lyapunov equation

$$
A_{c}^{T} P+P \cdot A_{c}=-Q,
$$

with $Q$ being a positive definite symmetric matrix. In the subsequent derivation, we will choose $Q$ such that

$\lambda_{\text {min }}(Q)>1$ with $\lambda_{\text {min }}(Q)$ being the minimum eigenvalue of $Q$. Define

$$
V_{M}=2^{-1} \lambda_{\min }(P)\left(M_{X}-\left\|Y_{r}\right\|_{\infty}\right)^{2},
$$

where $M_{x}$ is a positive constant and $Y_{r}=\left[y_{r}, \dot{y}_{r}, \ldots, y_{r}^{(n-1)}\right]^{T}$. Note that, if $\|x\| \geq M_{x} \geq\left\|Y_{r}(t)\right\|_{\infty}=\sup _{t \geq 0}\left\|Y_{r}(t)\right\|$, from (14), we have

$$
\begin{aligned}
V_{E} & \geq 2^{-1} \cdot \lambda_{\min }(P) \cdot\|E\|^{2} \\
& \geq 2^{-1} \cdot \lambda_{\min }(P) \cdot\left(\|x\|-\left\|Y_{r}(t)\right\|\right)^{2} \\
& \geq 2^{-1} \cdot \lambda_{\min }(P) \cdot\left(M_{x}-\left\|Y_{r}(t)\right\|_{\infty}\right)^{2}=V_{M} .
\end{aligned}
$$

Hence, if $V_{E}<V_{M}$, we have that $\|x\|<M_{x}$. Moreover, the derivative of $V_{E}$ along the trajectories of the closed-loop system (11) satisfies

$$
\begin{aligned}
\dot{V}_{E}= & 2^{-1} E^{T}\left(A_{c}^{T} P+P A_{c}\right) E \\
& +E^{T} P B_{c} \cdot\left[\widehat{f}-f+(\widehat{g}-g) u_{C}-g \cdot u_{S}-d_{0}\right] \\
\leq & -2^{-1} E^{T} Q E+\left|E^{T} P B_{c}\right| \\
& \times\left[|\widehat{f}|+|f|+\left|\widehat{g} u_{C}\right|+\left|g u_{C}\right|+d_{U}\right]-E^{T} P B_{c} g u_{S} .
\end{aligned}
$$

From (16), if there exists a supervisory control law with

$$
\begin{aligned}
& u_{S} \\
& =I^{*} \operatorname{sgn}\left(E^{T} P B_{c}\right) g_{L}^{-1}\left(f_{U}+\left|g_{U} u_{C}\right|+|\hat{f}|+d_{U}+\left|\widehat{g} u_{C}\right|\right),
\end{aligned}
$$

where $f_{U}, g_{L}, g_{U}$ are boundary functions for $f$ and $g$ such that $0 \leq|f| \leq f_{U}$ and $0<g_{L} \leq g \leq g_{U}$, then we can guarantee that

$$
\dot{V}_{E}<0, \quad \text { where } I^{*}= \begin{cases}1, & \text { for } V_{E} \geq V_{M}, \\ 0, & \text { otherwise. }\end{cases}
$$

For the following deviation, we define the modeling error

$$
e_{\text {mod }}=\widehat{f}\left(\theta_{f}^{*}\right)-f+\left[\widehat{g}\left(\theta_{g}^{*}\right)-g\right] u_{C}-d_{0},
$$

where $\theta_{f}^{*}$ and $\theta_{g}^{*}$ are the optimal parameters. Then (11) can be rewritten using Taylor series expansions as

$$
\begin{aligned}
\dot{E}=A_{c} E & -B_{c} g u_{S} \\
+ & B_{c}\left\{\widehat{f}\left(\theta_{f}\right)-\widehat{f}\left(\theta_{f}^{*}\right)+\left[\widehat{g}\left(\theta_{g}\right)-\widehat{g}\left(\theta_{g}^{*}\right)\right] u_{C}\right. \\
& \left.+e_{\text {mod }}\right\} .
\end{aligned}
$$

We have

$$
\begin{aligned}
& \widehat{f}\left(\theta_{f}\right)-\widehat{f}\left(\theta_{f}^{*}\right)=\left(\theta_{f}-\theta_{f}^{*}\right)^{T} \frac{\partial \hat{f}\left(\theta_{f}\right)}{\partial \theta_{f}}+\varepsilon_{f}, \\
& \widehat{g}\left(\theta_{g}\right)-\widehat{g}\left(\theta_{g}^{*}\right)=\left(\theta_{g}-\theta_{g}^{*}\right)^{T} \frac{\partial \widehat{g}\left(\theta_{g}\right)}{\partial \theta_{g}}+\varepsilon_{g},
\end{aligned}
$$

with $\varepsilon_{f}$ and $\varepsilon_{g}$ being the approximation errors of higher order terms. Now consider another Lyapunov function candidate $V_{G}$ given by

$$
\begin{aligned}
V_{G}= & 2^{-1} E^{T} P \cdot E+\left(2 \gamma_{f}\right)^{-1}\left(\theta_{f}-\theta_{f}^{*}\right)^{T}\left(\theta_{f}-\theta_{f}^{*}\right) \\
& +\left(2 \gamma_{g}\right)^{-1}\left(\theta_{g}-\theta_{g}^{*}\right)^{T}\left(\theta_{g}-\theta_{g}^{*}\right) .
\end{aligned}
$$

Using (20)-(22), we have

$$
\begin{aligned}
\dot{V}_{G}= & -2^{-1} E^{T} Q \cdot E+\gamma_{f}^{-1} \cdot\left(\theta_{f}-\theta_{f}^{*}\right)^{T} \\
& \times\left[\dot{\theta}_{f}+\gamma_{f} E^{T} P \cdot B_{c} \frac{\partial \widehat{f}\left(\theta_{f}\right)}{\partial \theta_{f}}\right] \\
& +\gamma_{g}^{-1} \cdot\left(\theta_{g}-\theta_{g}^{*}\right)^{T}\left[\dot{\theta}_{g}+\gamma_{g} E^{T} P \cdot B_{c} \frac{\partial \hat{g}\left(\theta_{g}\right)}{\partial \theta_{g}} u_{C}\right] \\
& -g \cdot E^{T} P \cdot B_{c} u_{S}+E^{T} P \cdot B_{c} \cdot\left[e_{\bmod }+\varepsilon_{f}+\varepsilon_{g} u_{C}\right] .
\end{aligned}
$$

Furthermore, as $E(0), e_{\bmod }(0), u_{C}(0), d_{0}, \varepsilon_{f}$ and $\varepsilon_{g}$ are bounded, and the projection method of the adaptation laws 


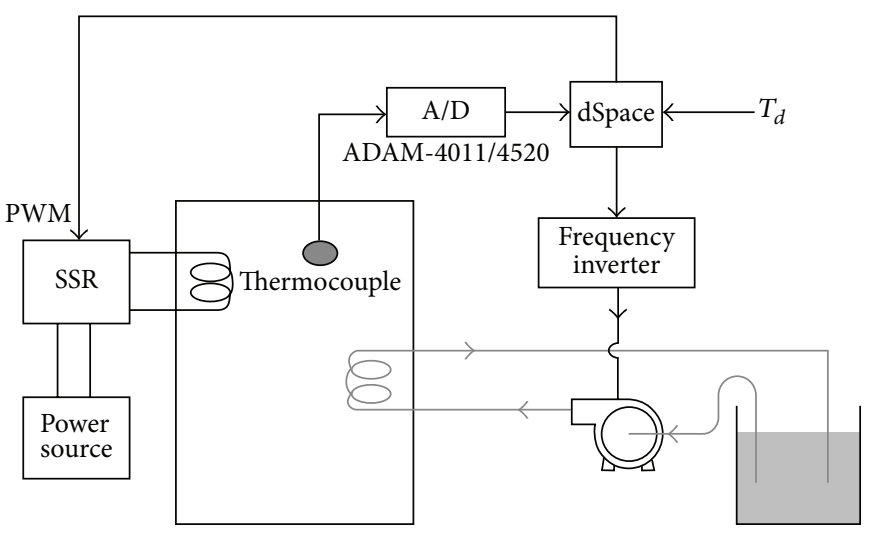

(a)

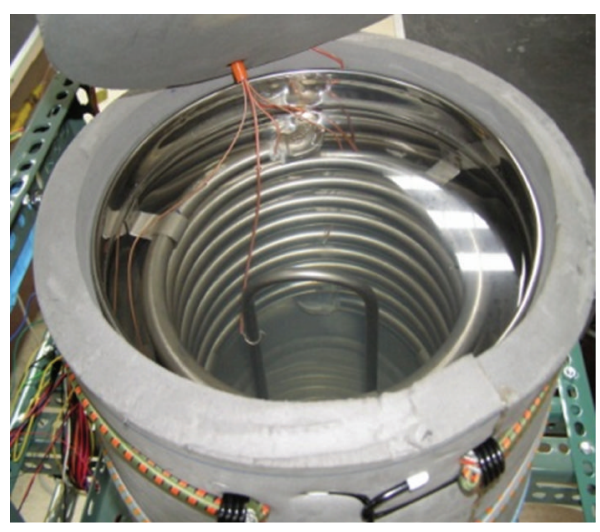

(b)

Figure 1: (a) Structure of the experimental setup. (b) A close view of the installation of heater, cooling pipe, and thermocouples in the water tank (with the cover being opened for observation). A $60 \mathrm{~mm}$ thick LDPE (low density polyethylene) foam insulates the tank and forms its cover.

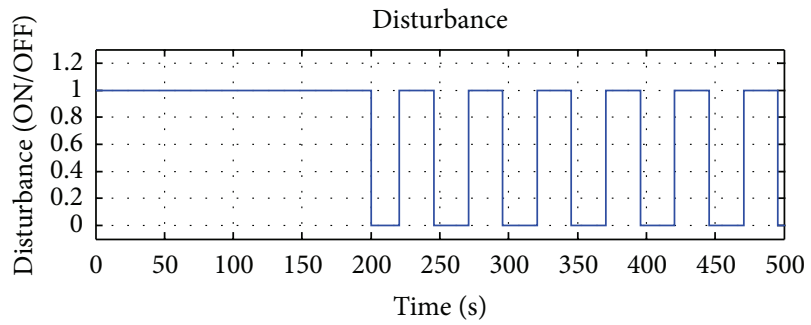

(a)

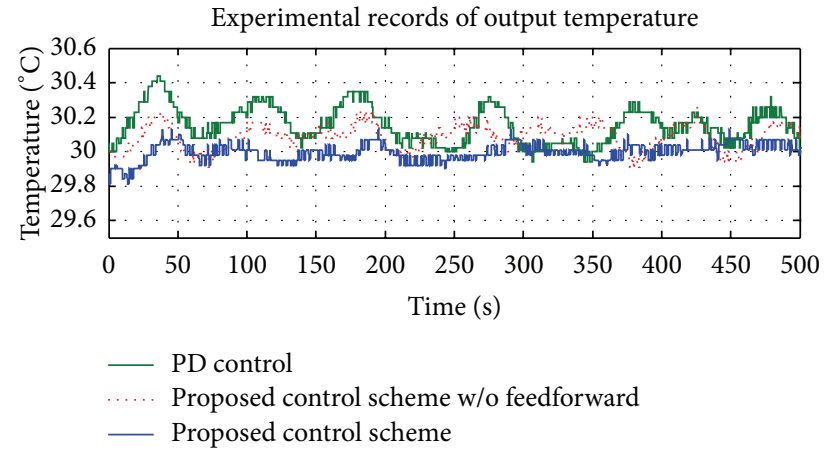

(b)

FIGURE 2: Experimental records of temperature when the system was controlled by four different control schemes.

ensures the boundedness of $u_{C}$, there exists a boundary function $\widetilde{e}$ such that

$$
\begin{aligned}
& \left|E^{T} P \cdot B_{c} \cdot\left[e_{\bmod }+\varepsilon_{f}+\varepsilon_{g} u_{C}\right]\right| \leq \widetilde{e}, \\
& \dot{V}_{G} \leq-2^{-1} E^{T} Q E+\left|E^{T} P B_{c}\left[e_{\bmod }+\varepsilon_{f}+\varepsilon_{g} u_{C}\right]\right| \\
& \quad \leq-2^{-1} \lambda_{\min }(Q)\|E\|^{2}+\widetilde{e} .
\end{aligned}
$$

This guarantees that $\dot{V}_{G}<0$, if $\|E\|>\left(\sqrt{\widetilde{e}} / \sqrt{2^{-1} \lambda_{\min }(Q)}\right)$. From the two adaptation laws (6) and (7), we obtain that if (24) is satisfied, the system (1) is uniformly ultimately bounded (UUB) stable.

\section{Experimental Study}

Temperature control systems are among the nonlinear systems with significant delay effects.

The proposed control scheme has been implemented on a prototype temperature control system. The system includes a water tank, a water pump, a resistor heater which serves as disturbance, and four thermal couples, as shown in Figure 1. The $40 \mathrm{~cm}$ diameter tank is filled with water to a depth of $60 \mathrm{~cm}$, the pump is driven by a $370 \mathrm{~W}$ frequency inverter, and the heater is driven by a SSR power IC. The control objective is to maintain the water temperature around the desired value $y_{r}=30^{\circ} \mathrm{C}$.

The function and gains of the supervisory control law (17) are given as $f_{U}(X)=1+0.004 x_{2}^{2}, g_{L}=2, g_{U}=2.2$. Moreover, we choose $k_{0}=0.3$ and $k_{1}=0.01$ so that the roots of $s^{2}+k_{1} s+$ $k_{0}=0$ are in the left-half complex plane. For (11), we have $A_{c}=\left[\begin{array}{cc}0 & 1 \\ -0.3 & -0.01\end{array}\right]$ and $B_{c}=\left[\begin{array}{l}0 \\ 1\end{array}\right]$. Besides, with $Q=\left[\begin{array}{cc}20 & 0 \\ 0 & 20\end{array}\right]$ for (13), we have $P=\left[\begin{array}{cc}1.3003 \times 10^{3} & 33.3333 \\ 33.3333 & 4.3333 \times 10^{3}\end{array}\right]$.

In designing the supervisory control law, we choose $M_{X}=$ 0.25 for (14) and $M_{f}=4, M_{g}=4, \gamma_{f}=1.6 \times 10^{-3}$, and $\gamma_{g}=0.8 \times 10^{-6}$ for the adaptation laws (6) and (7).

For comparison, three different control schemes were implemented based on similar initial states around $x_{1}(0)=$ $\xi(0)=30^{\circ} \mathrm{C}$ and $x_{2}(0)=\dot{\xi}(0)=0$ :

(1) the PD controller, $u(t)=K_{P} e(t)+K_{D} \dot{e}(t)$, which was exhaustively tuned for best tracking performance to be with $K_{P}=0.3$ and $K_{D}=0.05$,

(2) the proposed control scheme without the feedforwarded disturbance term $w_{m}(t)$. That is, $K_{F 0}=$ $\cdots=K_{F L}=0$ in (5),

(3) the proposed control scheme. 
Experimental results, shown in Figure 2, demonstrate that, in the face of disturbances, the output fluctuation was within $30 \pm 0.42^{\circ} \mathrm{C}$ for (1), within $30 \pm 0.25^{\circ} \mathrm{C}$ for (2), and within $30 \pm 0.15^{\circ} \mathrm{C}$ using the proposed control scheme. It is clear that the proposed scheme was able to achieve accurate tracking performance in the face of measurable or predictable disturbance. Furthermore, under the condition of immeasurable disturbance, temperature of the adaptively controlled system, the control scheme of (2), suffered from larger fluctuation but is still better than that of the PD controlled system, demonstrating effectiveness of the adaptation for the nonlinear and delayed temperature control system.

\section{Conclusion}

We proposed a simple yet effective adaptive neural control scheme for delayed nonlinear systems. Experimental results validate its effectiveness and show that the feed-forward of disturbance, if available, can achieve further improvements. It is clear that the proposed scheme has an excellent regulation performance when compared with PD control law, and the feedforward term can achieve further improvements.

\section{Acknowledgments}

This paper was sponsored in part by Chang Gung University and the National Science Council, Taiwan, under Contract nos. NSC 100-2221-E-182-008 and NSC 101-2221-E-182-006.

\section{References}

[1] C.-W. Chen, "Modeling, control, and stability analysis for timedelay TLP systems using the fuzzy Lyapunov method," Neural Computing and Applications, vol. 20, no. 4, pp. 527-534, 2011.

[2] J. Aulbach, B. Gjonaj, P. M. Johnson, A. P. Mosk, and A. Lagendijk, "Control of light transmission through opaque scattering media in space and time," Physical Review Letters, vol. 106, no. 10, Article ID 103901, 4 pages, 2011.

[3] B. Yu, C.-Z. Xu, and M. Guo, "Adaptive forwarding delay control for VANET data aggregation," IEEE Transactions on Parallel and Distributed Systems, vol. 23, no. 1, pp. 11-18, 2012.

[4] P. Cortes, J. Rodriguez, C. Silva, and A. Flores, "Delay compensation in model predictive current control of a three-phase inverter," IEEE Transactions on Industrial Electronics, vol. 59, no. 2, pp. 1323-1325, 2012.

[5] H. Dong, Z. Wang, D. W. C. Ho, and H. Gao, "Robust Ho fuzzy output-feedback control with multiple probabilistic delays and multiple missing measurements," IEEE Transactions on Fuzzy Systems, vol. 18, no. 4, pp. 712-725, 2010.

[6] Z. Wang, Y. Liu, G. Wei, and X. Liu, "A note on control of a class of discrete-time stochastic systems with distributed delays and nonlinear disturbances," Automatica, vol. 46, no. 3, pp. 543-548, 2010.

[7] G. Orosz and G. Stépán, "Hopf bifurcation calculations in delayed systems with translational symmetry," Journal of Nonlinear Science, vol. 14, no. 6, pp. 505-528, 2004.

[8] Q. Guo and C. Li, "Hopf bifurcation of a delayed differential equation," International Journal of Bifurcation and Chaos, vol. 17, no. 4, pp. 1367-1374, 2007.
[9] W. Yu, J. Cao, and G. Chen, "Stability and Hopf bifurcation of a general delayed recurrent neural network," IEEE Transactions on Neural Networks, vol. 19, no. 5, pp. 845-854, 2008.

[10] Z. Shu, Z. Xiong, and Z. He, "The stability and Hopf bifurcation for a predator-prey system with discrete and distributed delays," in Proceedings of the $3 r$ International Conference on BioMedical Engineering and Informatics (BMEI '10), pp. 1277-1281, Yantai, China, October 2010.

[11] C.-T. Chen and S.-T. Peng, "Intelligent process control using neural fuzzy techniques," Journal of Process Control, vol. 9, no. 6, pp. 493-503, 1999.

[12] F.-J. Lin, W.-J. Hwang, and R.-J. Wai, "A supervisory fuzzy neural network control system for tracking periodic inputs," IEEE Transactions on Fuzzy Systems, vol. 7, no. 1, pp. 41-52, 1999.

[13] Y.-C. Chen and C.-C. Teng, "A model reference control structure using a fuzzy neural network," Fuzzy Sets and Systems, vol. 73, no. 3, pp. 291-312, 1995.

[14] C. Li and K.-H. Cheng, "Recurrent neuro-fuzzy hybrid-learning approach to accurate system modeling," Fuzzy Sets and Systems, vol. 158, no. 2, pp. 194-212, 2007.

[15] K. Tanaka, "An approach to stability criteria of neural-network control systems," IEEE Transactions on Neural Networks, vol. 7, no. 3, pp. 629-642, 1996.

[16] Z.-R. Tsai, Y.-Z. Chang, J.-D. Hwang, and J. Lee, "Robust fuzzy stabilization of dithered chaotic systems using island-based random optimization algorithm," Information Sciences, vol. 178, no. 4, pp. 1171-1188, 2008.

[17] B. ZareNezhad and A. Aminian, "A multi-layer feed forward neural network model for accurate prediction of flue gas sulfuric acid dew points in process industries," Applied Thermal Engineering, vol. 30, no. 6-7, pp. 692-696, 2010.

[18] S. Patra, R. Jehadeesan, S. Rajeswari, and S. A. V. Satyamurthy, "Artificial neural network model for intermediate heat exchanger of nuclear reactor," International Journal of Computer Applications, vol. 1, no. 26, pp. 63-69, 2010.

[19] A. Vasickaninová, M. Bakošová, A. Mészáros, and J. J. Klemeš, "Neural network predictive control of a heat exchanger," Applied Thermal Engineering, vol. 31, no. 13, pp. 2094-2100, 2011.

[20] V. Rankovic, J. Radulovic, N. Grujovic, and D. Divac, "Neural network model predictive control of nonlinear systems using genetic algorithms," International Journal of Computers, Communications \& Control, vol. 7, no. 3, pp. 540-549, 2012.

[21] G. L. Plett, "Adaptive inverse control of linear and nonlinear systems using dynamic neural networks," IEEE Transactions on Neural Networks, vol. 14, no. 2, pp. 360-376, 2003.

[22] W.-D. Chang, R.-C. Hwang, and J.-G. Hsieh, "Application of an auto-tuning neuron to sliding mode control," IEEE Transactions on Systems, Man and Cybernetics Part C, vol. 32, no. 4, pp. 517$522,2002$. 


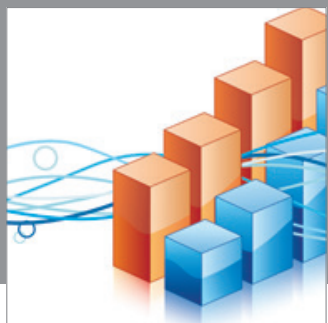

Advances in

Operations Research

mansans

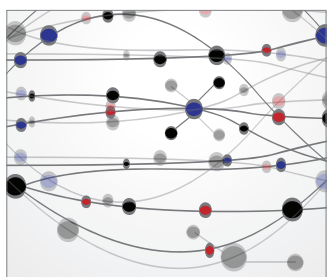

The Scientific World Journal
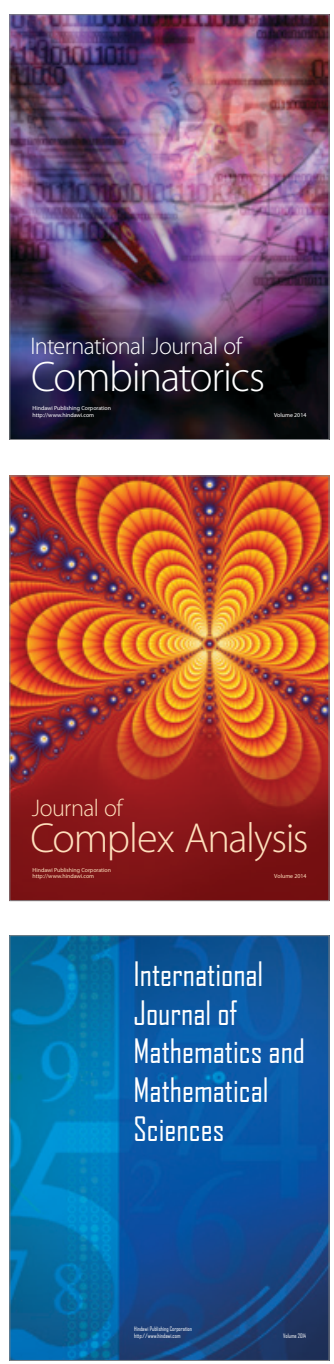
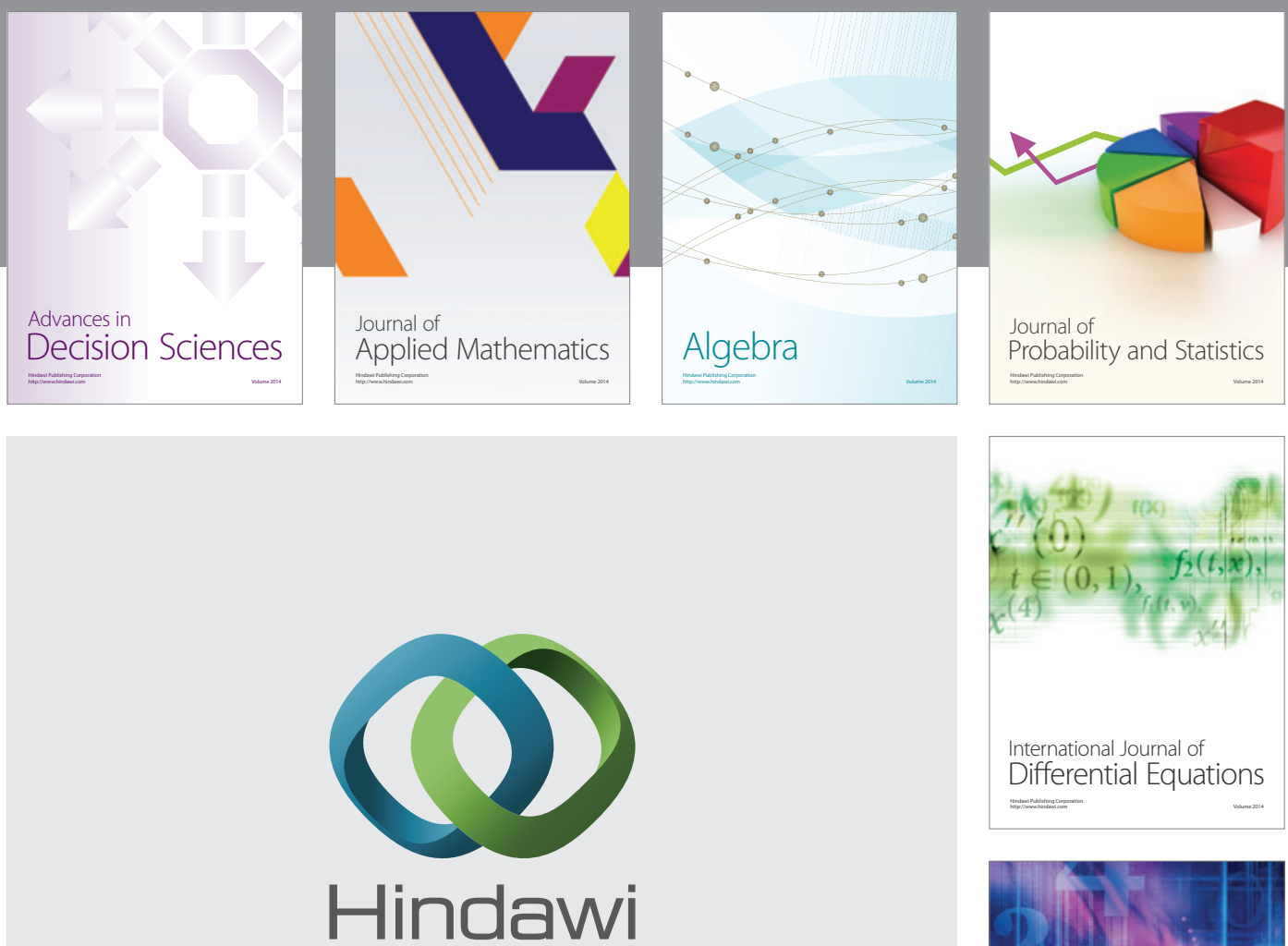

Submit your manuscripts at http://www.hindawi.com
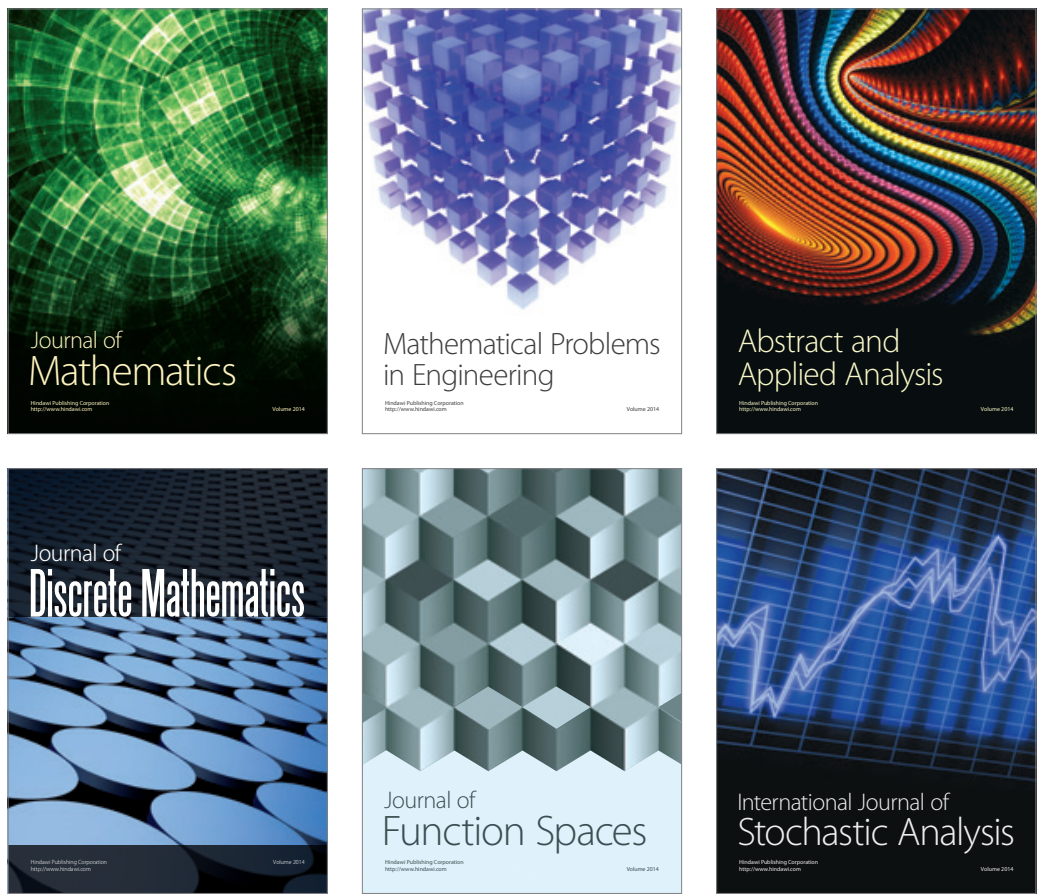

Journal of

Function Spaces

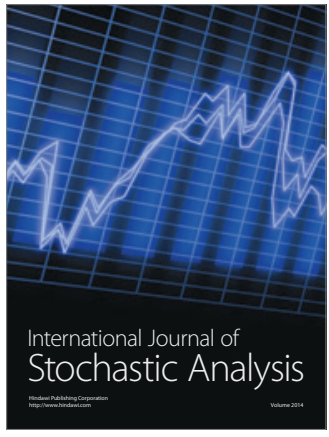

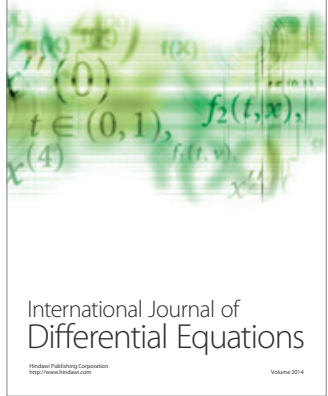
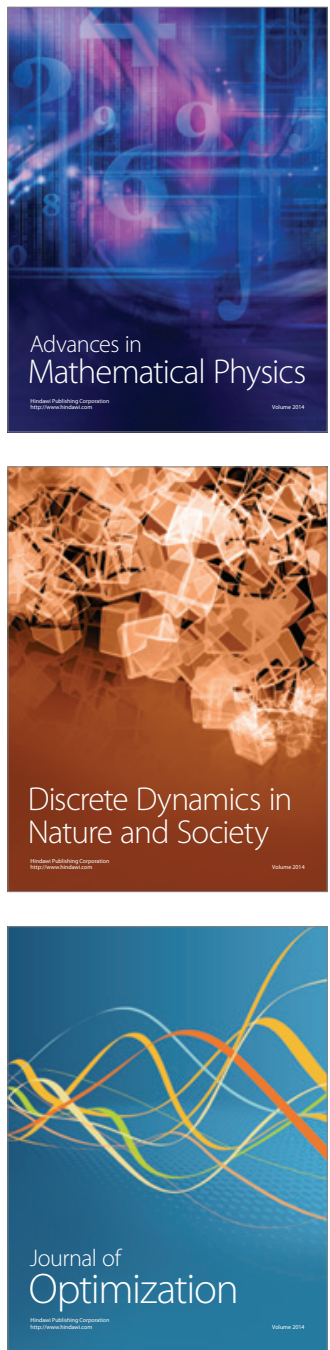